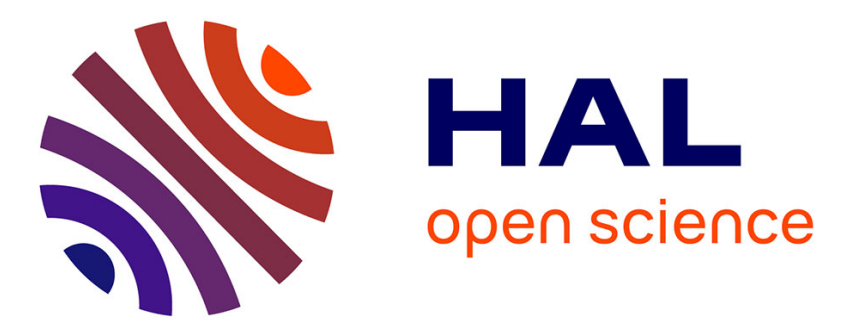

\title{
Perceptual Effect of Shoulder Motions on Crowd Animations
}

Ludovic Hoyet, Anne-Hélène Olivier, Richard Kulpa, Julien Pettré

\section{To cite this version:}

Ludovic Hoyet, Anne-Hélène Olivier, Richard Kulpa, Julien Pettré. Perceptual Effect of Shoulder Motions on Crowd Animations. ACM Transactions on Graphics, 2016, 35 (4), pp.1-10. 10.1145/2897824.2925931. hal-01357713

\section{HAL Id: hal-01357713 \\ https://inria.hal.science/hal-01357713}

Submitted on 30 Aug 2016

HAL is a multi-disciplinary open access archive for the deposit and dissemination of scientific research documents, whether they are published or not. The documents may come from teaching and research institutions in France or abroad, or from public or private research centers.
L'archive ouverte pluridisciplinaire HAL, est destinée au dépôt et à la diffusion de documents scientifiques de niveau recherche, publiés ou non, émanant des établissements d'enseignement et de recherche français ou étrangers, des laboratoires publics ou privés. 


\section{Perceptual Effect of Shoulder Motions on Crowd Animations}

\author{
Ludovic Hoyet* \\ Inria
}

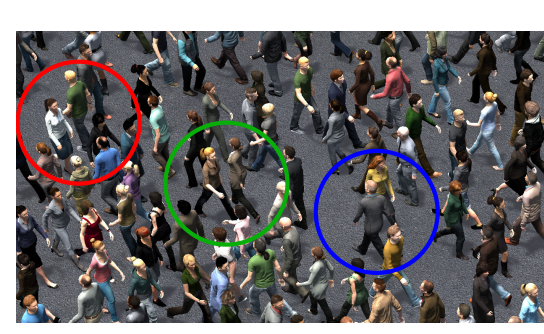

\author{
Richard Kulpa $a^{\ddagger}$ \\ Univ. Rennes 2
}

\author{
Julien Pettré \\ Inria
}
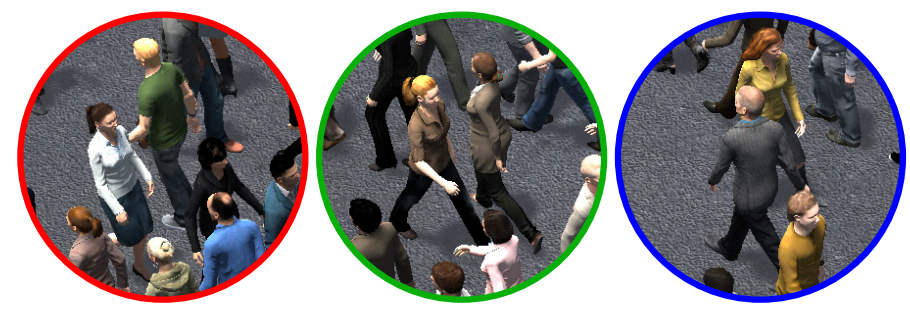

Figure 1: Crowd simulators typically do not account for character interaction influences at the animation level (red circle), while secondary motions such as shoulder motions can prevent spectators from perceiving slight residual collisions and globally increase the perceived level of animation naturalness (green and blue circles).

\begin{abstract}
A typical crowd engine pipeline animates numerous moving characters according to a two-step process: global trajectories are generated by a crowd simulator, whereas full body motions are generated by animation engines. Because interactions are only considered at the first stage, animations sometimes lead to residual collisions and/or characters walking as if they were alone, showing no sign to the influence of others. In this paper, we investigate the value of adding shoulder motions to characters passing at close distances on the perceived visual quality of crowd animations (i.e., perceived residual collisions and animation naturalness). We present two successive perceptual experiments exploring this question where we investigate first, local interactions between two isolated characters, and second, crowd scenarios. The first experiment shows that shoulder motions have a strong positive effect on both perceived residual collisions and animation naturalness. The second experiment demonstrates that the effect of shoulder motions on animation naturalness is preserved in the context of crowd scenarios, even though the complexity of the scene is largely increased. Our general conclusion is that adding secondary motions in character interactions has a significant impact on the visual quality of crowd animations, with a very light impact on the computational cost of the whole animation pipeline. Our results advance crowd animation techniques by enhancing the simulation of complex interactions between crowd characters with simple secondary motion triggering techniques.
\end{abstract}

Keywords: Human Animation, Crowd Simulation, Interactions, Perception

Concepts: •Computing methodologies $\rightarrow$ Motion capture; Collision detection; Perception;

\footnotetext{
*ludovic.hoyet@inria.fr

† anne-helene.olivier@univ-rennes2.fr

‡richard.kulpa@univ-rennes2.fr

$\S$ julien.pettre@inria.fr
}

Permission to make digital or hard copies of all or part of this work for personal or classroom use is granted without fee provided that copies are not made or distributed for profit or commercial advantage and that copies bear this notice and the full citation on the first page. Copyrights for components of this work owned by others than ACM must be honored. Abstracting with credit is permitted. To copy otherwise, or republish, to post on servers or to redistribute to lists, requires prior specific permission and/or a fee. Request permissions from permissions@acm.org. (C) 2016 ACM.

SIGGRAPH 2016, July 24-28, 2016, Anaheim, CA

\section{Introduction}

Animating crowds to populate digital worlds is a requirement for interactive applications. The beneficial effects of a lively background made of many moving characters in terms of visual quality and enjoyment is undeniable. The current trend is to create more impressive scenes, of a larger size, with a higher population density. This stresses the need for highly efficient methods to animate crowds where efficiency means the best possible trade-off between visual quality and computational efficiency.

We focus on the typical use of crowd engines to animate numerous walking characters. Such engines are typically based on a two-step pipeline [Thalmann 2007]. First, a crowd simulator generates the characters' global 2D displacement trajectories in the environment. Second, an animation system transforms these global trajectories into full body motions. This two-step decomposition is interesting for computational reasons. Crowd simulators raise quadratic complexity issues by nature. For the sake of simplicity, simulation models are often limited to 2D moving circles with 3 degrees of freedom (DoF), i.e., two translations and a rotation. The complete set of internal trajectories ( 30 to $60 \mathrm{DoF}$ per character) is then considered at the animation step only, where characters are processed independently. This two-step process avoids combining the complexity of crowd simulators with the dimensionality of character kinematic models, however this decomposition has drawbacks. The notion of interactions between characters is considered at the simulation level, and is lost at the animation level. Body animations are therefore not influenced by the presence of neighbours, only global trajectories are. Finally, discrepancies between the shapes of the 2D simulated agents and the animated geometrical character 3D models may create artefacts, such as residual collisions.

Is it possible to extend character animation techniques to break the impression of characters' passivity? Would that be a real benefit for the visual aspects of the resulting crowd animations? Is it possible to only change the animation step to avoid costly effects on computational performances? Would that overcome some limitations of the two-step decomposition of the crowd engine pipeline?

We explore these questions by perceptually investigating the effects of adding secondary motions to character walking animations. Effects are evaluated on visual quality that we probe in terms of animation naturalness and detection of residual collisions between characters. More specifically, we focus on shoulder motions, as

ISBN: 978-1-4503-4279-7/16/07

DOI: http://doi.acm.org/10.1145/2897824.2925931 
illustrated in Figure 1, with several goals in mind. First, we believe that shoulder motions will give the impression that characters achieve complex interactions, with a positive effect on the perceived quality of animations. In particular, we expect that these motions will make characters passing at close distances visually more natural, and might even prevent viewers from perceiving some of the residual collisions. Finally, we demonstrate that shoulder motions can be successfully added at the animation stage without increasing the complexity of the whole pipeline.

To understand these issues, we first focused on the local effect of these secondary motions and conducted a perceptual experiment considering two characters walking in opposite directions and passing by each other at close distances. In this context, our first contribution is to answer the following questions: does adding shoulder motions a) improve motion naturalness? b) prevent users from detecting residual collisions? The experimental factors we controlled were: the interpersonal distance separating their parallel trajectories and the addition of shoulder motions for none, one or both characters. We found that shoulder motions have strong positive effects on the visual quality of animations, where such animations are perceived to be significantly more natural, and residual collisions become significantly less perceptible.

Our second contribution is to perceptually evaluate the benefits of shoulder motions in the situation of crowded scenes, where shoulder motions are diluted into much more visually complex animations. We first designed simple shoulder motion triggering rules for crowd characters. We considered two kind of crowded situations and controlled the frequency of shoulder motion occurrences as well as crowd density. We asked again participants about their perception of residual collisions as well as naturalness of animations. Results show a significant positive effect of shoulder motions on the animation naturalness. This increase of visual quality is obtained at a very low computational overhead, which demonstrates the relevance of the direction explored by our work. The effect on residual collision is however lost, indicating that this criterion may be perceived by participants based on different cues in the context of crowds, which opens perspectives for future research.

\section{Related Work}

Crowd Simulation. Crowd simulators compute the global displacement of hundreds of characters based on the macroscopic motion of a global flow [Hughes 2003; Treuille et al. 2006] or based on local interactions between simulated agents. Many models of local interactions have been proposed: social forces [Helbing and Molnár 1995], flocking rules [Reynolds 1987], velocity-obstacles [van den Berg et al. 2008], synthetic vision [Ondřej et al. 2010], power law [Karamouzas et al. 2014], etc. The field has received a lot of attention, and many extensions of these models have been proposed that would be impossible to exhaustively overview here. It is however noticeable that some recent approaches focus on secondary motions humans perform to avoid collisions. In particular, the simplistic geometrical representation of agents, most often a $2 \mathrm{D}$ circle that can turn or move forwards approximately bounds the character, is reconsidered. Shoulders rotations are simulated based on overlapping circles [Korhonen et al. 2009] or capsules [Stüvel et al. 2016], or using additional kinematic information such as footprints to navigate characters in dynamic crowds [Singh et al. 2011]. Also, Hughes et al. [2014] experimentally evaluated sidestepping strategies to include in an holonomic model. While these approaches demonstrate that it is possible to include such strategies at the simulation step, such evolutions largely impact the performance of the simulation step since the dimension of agent kinematics models is increased and geometrical simplicity is partially lost.
Crowd Characters Animation. Crowd simulators generate global (root) trajectories for characters. The animation component is then in charge of synthesizing the corresponding whole body motion. Motion capture-based techniques are generally used in such tasks as they provide high quality animations at low computational cost, a crucial criterion in the context of crowds. Two different situations are generally considered: static (e.g., groups of conversing characters [Ennis and O'Sullivan 2012]) and walking characters. Walking characters are more costly to animate because they require dedicated techniques, such as using motion blending techniques to ensure controllability: walking velocity can be continuously adapted so that characters can accurately follow the guide trajectory [Rose et al. 1998; Lee et al. 2002; Kwon and Shin 2005; Treuille et al. 2007]. This procedural technique however tends to generate uniform animations. To increase variety, one idea is to add secondary motions, such as by editing limb positions [Maïm et al. 2009]. Resultantly characters also look unresponsive, as those dedicated techniques do not consider on-going interactions between characters.

Crowd animations can also be fully generated from motion capture data that capture interactions between characters [Shum et al. 2008; Kim et al. 2012]. Such approaches are not based on the common combination of simulation and animation techniques considered in our paper, and used in most interactive applications. Finally, character meshes can also be used to test for residual collisions after animation is performed, but such techniques are still too computationally expensive for interactive applications, even if some approaches try to simplify the problem, e.g., by hierarchically approximating the characters' shape [Stüvel et al. 2014].

Perceptual Evaluation of Crowd Animations. Animating large crowds for interactive applications such as video games requires eradicating any useless computational expense. Perceptual studies are therefore beneficial to evaluate the importance of simplifications made to the simulation or animation techniques on the visual quality of results [Reitsma and O'Sullivan 2009; Yeh et al. 2009], in order to find optimal trade-offs between complexity and visual accuracy. It was for example used to determine the required number of visual and motion templates [McDonnell et al. 2008], the importance of synchronising characters during interactions [Ennis et al. 2010], or the thresholds producing physically plausible interactions between characters [Hoyet et al. 2012]. In particular, it has been demonstrated that collision avoidance algorithms can be progressively discarded for background characters [Kulpa et al. 2011].

Contribution. In this paper, we demonstrate through perceptual experiments that shoulder motions can be introduced at the animation stage to improve crowd animations. Unlike previous techniques which try to introduce shoulder motions at the simulation stage (e.g., [Korhonen et al. 2009; Stüvel et al. 2016]), we believe that keeping untouched the complexity of the simulation stage can be more relevant (same simplistic agent geometrical and interaction models). We believe that introducing secondary motions due to interactions between characters at the animation stage would improve the visual quality of resulting crowd simulations without complexifying the already computationally expensive simulation stage. In order to demonstrate the benefits of our approach, we designed two perceptual studies, exploring first local interactions between two characters only (Section 3), then larger-scale scenarios (Section 4).

\section{Perceptual Effect of Shoulder Motions in Two-Character Interactions}

The general goal of this experiment is to evaluate the contribution of adding shoulder motions to the visual quality of walking interactions. We first study this effect at the local scale, and therefore consider the situation of interactions between two characters walk- 

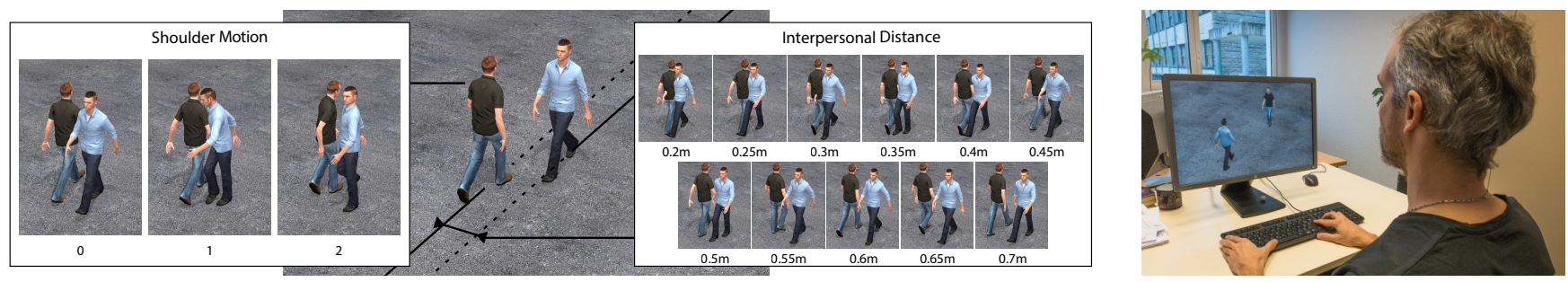

Figure 2: Overview of the first experiment. (Left) We presented to participants videos of two characters walking past each other at distances ranging from 0.2 to $0.7 \mathrm{~m}$. Either none of the characters, one character or both characters were displaying shoulder motions. (Right) Experimental setup and participant in the experiment.

ing in opposite directions and passing at close distances. In this situation, the questions which guide the design of this experiment are the following. First, when interaction distance between characters is not sufficient to prevent residual collisions, does adding shoulder motions have an effect on how spectators perceive such residual collisions? Second, does adding shoulder motions have an effect on the resulting naturalness of animations?

\subsection{Material and Methods}

\subsubsection{Pre-experiment}

Capturing human interactions involving shoulder motions to avoid collisions raises the question of whether one or multiple strategies are naturally displayed by humans. One is whether people preferentially present their torso or their back in close avoidance conditions. As this difference is important in experiments such as the one presented in this paper, we conducted a short informal study where we asked pairs of participants to walk past each other in a small corridor, which forced them to display naturally such shoulder strategies. Out of 9 participants tested, 7 consistently presented their torso, while only 1 presented his back and one never displayed shoulder rotations but always forced his way through.

\subsubsection{Motion Capture}

For the purpose of this study, two pairs of male actors, tested to consistently display a torso strategy, participated in a motion capture session. For each motion capture set, we recorded two actors walking past each other at a distance of $1.5 \mathrm{~m}$ (4 trials) and $40 \mathrm{~cm}(12$ trials). In the $40 \mathrm{~cm}$ situation, we captured 3 conditions $(3 \times 4$ trials): 1) only the first actor avoided the collision, 2) only the second actor avoided, and 3) both actors avoided. Actors were instructed to avoid each other as naturally as possible using shoulder motions. They were asked to deviate as little as possible from their trajectory marked by lines on the floor. They were finally asked to keep walking straight when not instructed to avoid the other actor. The two distances were chosen so that actors were sufficiently far apart in the $1.5 \mathrm{~m}$ condition to ensure that there was absolutely no shoulder avoidance reaction necessary, and on the contrary that for the $40 \mathrm{~cm}$ condition a shoulder motion would be required by at least one of the actors without necessitating a deviation of trajectory.

Motion capture was conducted using a 14-camera Vicon optical system, with a setup allowing us to capture accurately an area of $6 \mathrm{~m} \times 6 \mathrm{~m}$. Body motion was captured at $120 \mathrm{~Hz}$ using 57 markers placed on standardized landmarks on the body of each actor, then mapped onto a skeleton where joint angles were computed and used to drive the virtual characters in Autodesk 3dsMax. Two virtual characters were chosen for creating the experiment stimuli (see Figure 2). Note that prior to the motion capture session we took care of selecting actors who roughly matched the height of those characters to minimize motion retargeting errors.

\subsubsection{Factors}

Shoulder Motion. We were interested in whether shoulder motions could be used to mask collisions in paired interactions, using three conditions: none, one or both characters displayed shoulder motions (resp. labelled Shoulder Motion: 0, 1 and 2).

Interpersonal Distance. Depending on the crossing distance between two walkers, we can obviously expect that shoulder motions will be more or less relevant in the collision avoidance behaviour. We selected 11 interpersonal distances, ranging from $0.2 \mathrm{~m}$ to $0.7 \mathrm{~m}$ by $0.05 \mathrm{~m}$ steps. This interpersonal distance was computed as the distance between the root of the characters at the time of interaction, i.e., when the distance between characters is minimal.

Interpenetration Volume. While interpersonal distance is a relevant and easy-to-control parameter for crowd simulation, shoulder motions naturally reduce the distance where collisions start to occur, because of the modification of the interpersonal volume between characters. Exploring the perception of collisions regarding the actual volume of interpenetration can be therefore more relevant from a perceptual point of view. This factor will be considered a posteriori of the experiment. For every frame of every clip observed by participants, we computed the volume of interpenetration, based on the intersection volume of the characters' mesh (using the intersection boolean operation in 3dsMax). To compare volumes of interpenetration in a linear way between clips, we then expressed the volume $V_{i}$ of each clip $i$ as:

$$
V_{i}=\frac{1}{n_{i}} \sum_{f=1}^{N} \sqrt[3]{v_{i}(f)}
$$

where $v_{i}(f)$ is the interpenetration volume (in $\mathrm{m}^{3}$ ) at frame $f$ of clip $i$ (out of $N$ frames), and $n_{i}$ is the number of frames where $v_{i}(f) \neq 0$ is non-null (i.e., when there is a collision). Figure 3 displays the relation between Interpersonal Distance and Interpenetration Volume computed for the stimuli used in the present study.

\subsubsection{Stimuli Creation}

We created stimuli by combining the 3 Shoulder Motions with the 11 Interpersonal Distances. Motions of non-avoiding characters were selected from the $1.5 \mathrm{~m}$ trials (out of 16 motion clips), to ensure that non-avoiding characters did not display any avoidance reaction, even as little as getting an arm slightly out of the way. Motions of avoiding characters were selected from the $40 \mathrm{~cm}$ trials, out of 32 motion clips. When more than one character displayed shoulder motions, we also ensured that both motions were selected from different motion capture trials to avoid a potential confounding factor. To synchronise motion capture clips, we computed for each motion capture trial the time when interaction occurred, defined as the frame with the minimum distance between the root of the ac- 


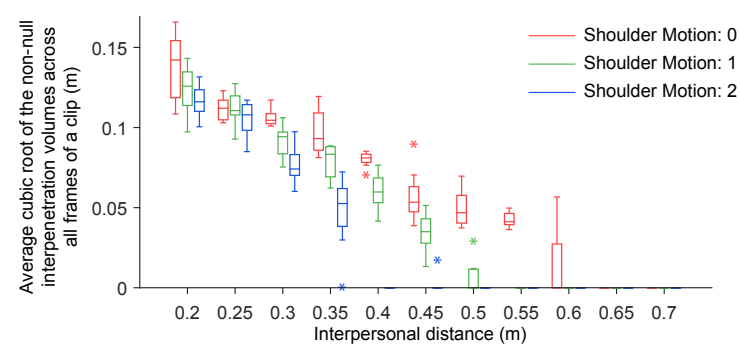

Figure 3: Boxplot of the interpenetration volume for each Shoulder Motion $\times$ Interpersonal Distance condition.

tors' skeleton. Motion capture clips from different trials were then aligned in time based on their respective time of interaction.

To avoid a particular motion clip to influence the results, we created 8 video clips (repetitions) for each Shoulder Motion $\times$ Interpersonal Distance condition. In half of the repetitions, characters were crossing on their left side, and we selected the avoiding motion clips accordingly to present the torso on the correct side. Each character was selected to start at the top right of the screen in half of the repetitions, and at the bottom left in the other half.

Previous work demonstrated that the perception of collisions in such interactions is highly influenced by the camera viewpoint [Kulpa et al. 2011]. As our goal is not to evaluate the specific effect of camera viewpoint, we selected a canonical viewpoint providing as much information as possible about the interaction (Figure 2), which is also a viewpoint commonly used in video games.

\subsubsection{Apparatus and Procedure}

Participants were presented in random order with a total of 264 video clips: 3 Shoulder Motion $(0,1,2) \times 11$ Interpersonal Distance $(0.2 \mathrm{~m}$ to $0.7 \mathrm{~m}$ with a $0.05 \mathrm{~m}$ step $) \times 8$ repetitions. Each video clip lasted from 3 to $4 \mathrm{~s}$. Participants were comfortably seated at their preferred distance from a 24-inch screen (approx. $60 \mathrm{~cm}$ ), and all the videos were displayed at $1920 \times 1200$ pixels and at $60 \mathrm{~Hz}$ using Psychophysics Toolbox v3 for Matlab. To get used to the stimuli, six examples that were not seen afterwards (additional video clips) were presented in random order to participants at the beginning of the experiment: 3 Shoulder Motion $(0,1,2) \times 2$ Interpersonal Distance $(0.2 \mathrm{~m}, 0.7 \mathrm{~m})$. We chose a 2-Alternative Forced Choice protocol (2AFC): after each trial, participants were asked "Did the characters go through each other?" (yes/no answer using the keyboard). They were then asked to rate how natural they considered the interaction to be on a scale from 1 (not very natural) to 7 (very natural) using the keyboard.

Fifteen participants volunteered for this experiment $(2 \mathrm{~F}, 13 \mathrm{M}$; avg. age: $27.7 \pm 3.7$, range: 24 to 37 ). They were recruited via internal mailing lists amongst students and staff, and were all naïve with respect to the purpose of the experiment. All had normal or corrected-to-normal vision. They gave written and informed consent and the study conformed to the declaration of Helsinki.

\subsection{Analysis}

\subsubsection{Dependent variables}

Perceived collisions. We computed the proportion of perceived collisions across repetitions for each participant depending on the studied factors. To relate interpersonal distance and interpenetration volume to the perception of collision, we also fitted for each Shoulder Motion condition a logistic psychometric function to the

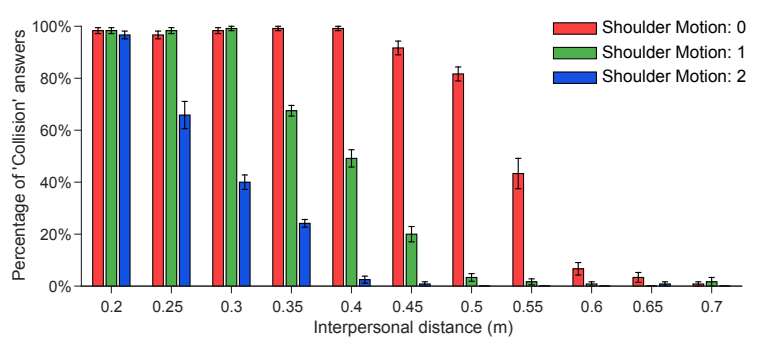

Figure 4: Proportion of perceived collisions ( \pm SEM) for each Shoulder Motion $\times$ Distance condition.

results of each participant, using the Psychometric Toolbox for Matlab. The psychometric curve is a mathematical model representing how the observer's response to the stimuli varies depending on the variation of these stimuli. It allows us to calculate the critical threshold, representing the critical interpersonal distance $D_{\text {crit }}$ and the critical interpenetration volume $V_{\text {crit }}$ where participants consider $50 \%$ of the time that there is a collision between characters.

Naturalness ratings. We evaluated participants' naturalness ratings of interactions by computing the average of participants' answer over repetitions for each participant.

\subsubsection{Statistical Analysis}

Normality was assessed using a Kolmogorov-Smirnov test. All effects were reported at $p<0.05$. To assess whether Shoulder Motion and Interpersonal Distance affected the percentage of perceived collisions and naturalness ratings, we performed separated two-way repeated measures Analysis of Variance (ANOVA) on these withinsubjects factors. We then assessed the influence of Shoulder Motion on $D_{c r i t}$ and $V_{c r i t}$, and performed separated one-way repeated measure ANOVA with this factor. Effect size was computed using partial eta squared $\left(\eta_{p}^{2}\right)$. Greenhouse-Geisser adjustments to the degrees of freedom were applied when appropriate to avoid any violation of the sphericity assumption. When we found main or interaction effects, we further explored the cause of these effects using Bonferroni post-hoc tests for pairwise comparisons. In order to relate naturalness ratings with perceived collisions, we computed correlations between these data sets across participants. All the significant effects are summarized in Table 3.

\subsection{Results}

Perceived collisions. Regarding the first Analysis (Table 3.A), we found a main effect of Interpersonal Distance, showing that participants perceive on average less collisions when the distance between characters increases (until $0.6 \mathrm{~m}$ after which almost no collisions are perceived). We also found a main effect of Shoulder Motion, where participants perceive on average less collisions when characters depict shoulder reactions (less collisions perceived on average when 2 characters avoid than 1, then 0). Finally, we found a Shoulder Motion $\times$ Interpersonal Distance interaction effect, showing that the range of distances for which participants perceive collisions decrease with shoulder reactions (Figure 4). Collisions were perceived in more than $90 \%$ of the cases from $0.2 \mathrm{~m}$ to $0.45 \mathrm{~m}$ (no shoulder motion), $0.2 \mathrm{~m}$ to $0.3 \mathrm{~m}$ (one character displaying shoulder motions), and only for $0.2 \mathrm{~m}$ (both characters displaying reactions).

To evaluate the effect of both interpersonal distance and interpenetration volume on the perception of collisions, we then computed the critical thresholds $D_{\text {crit }}$ and $V_{\text {crit }}$ for all participants, and performed 2 one-way ANOVAs (factor: Shoulder Motion, results in Table 3.B and 3.C). In both cases, we found a main effect of Shoulder Motion. Post-hoc analyses showed that $D_{\text {crit }}$ was significantly 

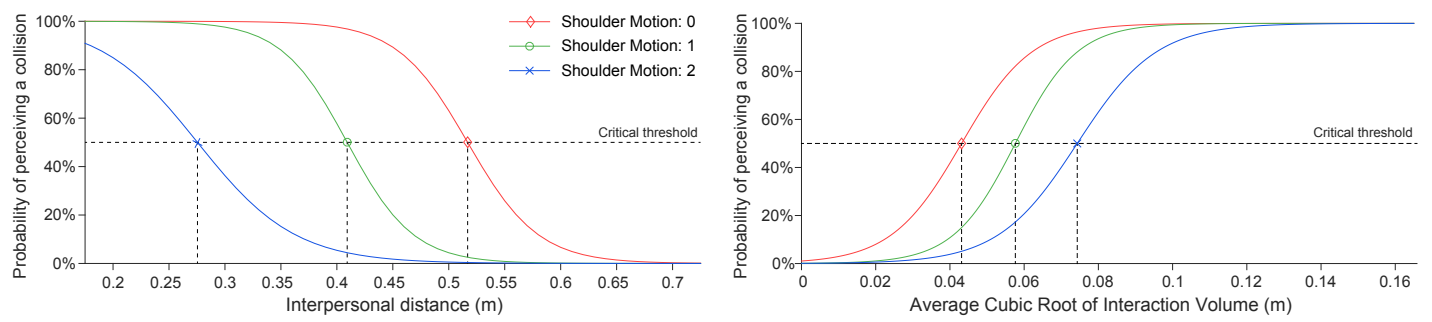

Figure 5: Psychometric curves depicting for each Shoulder Motion condition the probability of perceiving a collision depending on (Left) the distance between characters' roots and (Right) the average cubic root of the interaction volume between characters.
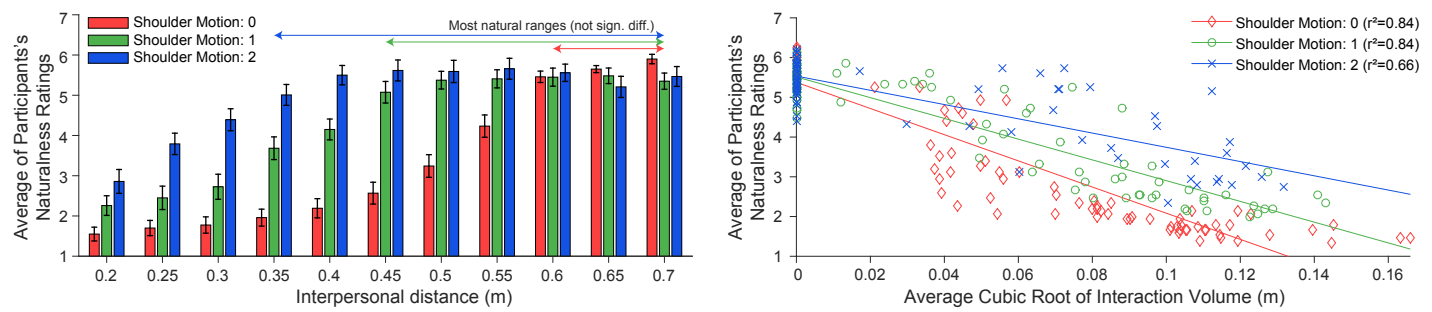

Figure 6: Average ( $\pm S E M)$ naturalness ratings (over all participants) of each Shoulder Motion condition: in relation to (Left) interpersonal distance and (Right) interpenetration volume.

lower and $V_{\text {crit }}$ significantly higher when both, then one, then none of the characters displayed shoulder motions. Therefore, the critical distance where participants are unsure if a collision happens is decreased when characters display shoulder motions. Similarly, the critical volume of interpenetration increases when shoulder motions are presented (Figure 5). Average critical thresholds across participants are summarized in Table 1.

\begin{tabular}{cccc} 
Shoulder Motion & 0 & 1 & 2 \\
\hline$D_{\text {crit }}(\mathrm{m})$ & $0.52 \pm 0.02$ & $0.41 \pm 0.01$ & $0.27 \pm 0.03$ \\
\hline$V_{\text {crit }}(\mathrm{m})$ & $0.043 \pm 0.007$ & $0.058 \pm 0.004$ & $0.075 \pm 0.008$ \\
\hline
\end{tabular}

Table 1: Average $( \pm S D)$ of the critical distance and volume thresholds for each Shoulder Motion condition.

Naturalness ratings. There was a main effect of Shoulder Motion on naturalness ratings (Table 3.D): participants perceive interactions where characters display shoulder motions to be on average more natural (with average naturalness increasing with the number of characters displaying such reactions). There was also a main effect of Interpersonal Distance: on average naturalness increases with interpersonal distance until $0.55 \mathrm{~m}$ where it reaches a plateau. Finally, a Shoulder Motion $\times$ Interpersonal Distance interaction effect showed that naturalness ratings reach their maximum value for lower interpersonal distances when more characters display shoulder motions. Ranges of distances significantly more natural are (see Figure 6, Left): over 0.35m (Shoulder Motion 2), over $0.45 \mathrm{~m}$ (Shoulder Motion 1) and over 0.6m (Shoulder Motion 0).

We also looked at the relation between naturalness and interpenetration volume. Figure 6 (Right) shows participants' average naturalness ratings for the different interpenetration volumes of each Shoulder Motion condition. We performed linear regressions on this data for each subject and condition, then performed a repeated measure ANOVA with within-subjects factor Shoulder Motion on the y-intercept and slope values of the linear regressions. We did not find a significant effect for $y$-intercept values, meaning that shoulder motions do not seem to influence the naturalness of interactions when no collision occurs. However, we did find a significant effect of Shoulder Motion on slope values (Table 3.E), where post-hoc analysis demonstrated that for the same interpenetration volume, interactions are rated to be significantly more natural when more characters display shoulder reactions. We also looked at correlations between naturalness ratings and collisions answers for all participants, and found negative correlations for each Shoulder Motion condition $(r=-0.90, r=-0.80$ and $r=-0.64$; all $p \approx 0)$.

\subsection{Discussion}

In the context of two characters passing by each other at close distances, these results demonstrate that shoulder motions improve how viewers perceive interactions between characters. Shoulder motions decrease the overall probability of perceiving collisions, influence the range of distances between characters where collisions are perceived by viewers, as well as the residual volume of interpenetration tolerated in interactions where collisions are not detected by viewers. They also improve the naturalness of interactions, which was found to be negatively correlated with the proportion of perceived collisions.

In this experiment, we directly controlled the interpersonal distance between both characters, a parameter that is commonly and easily controllable in crowd simulation algorithms. Such algorithms often approximate characters using 2D circles, whose radius directly influences the minimum interpersonal distance between characters. Wide circles overestimate the characters' shape to prevent any residual collisions, but also prevent simulations from reaching high densities, while narrow ones result in numerous residual collisions between the final animated meshes. Our results suggest that shoulder motions, considered at the animation step, could be used with smaller 2D circle radii (simulation step), to reduce the amount of residual collisions perceived by viewers and increase the naturalness of interactions. Of course, these results hold up to a certain minimum interpersonal distance, where collisions become too obvious and cannot be masked anymore, even with shoulder motions.

While interpersonal distance is usually used to control interactions, collisions between characters are actually characterised by the volume of interpenetration between the characters' mesh. We found that shoulder motions increase the critical volume of interpenetration tolerated by viewers, and therefore, such reactions indeed manage to hide larger residual collisions. However, interpenetration volumes are still too computationally expensive to be used in inter- 
active applications, even if more relevant from a perceptual point of view. Evaluating if select parts of the characters' body are more specifically used by viewers to detect interpenetrations (e.g., are users looking more at the lower or upper body to detect collisions?) could help simplifying such computations.

In conclusion, two-character situations were used to explore the local mechanisms involved in the perception of such interactions. These results provide valuable insights which will be integrated and validated on larger-scale scenarios in the following section.

\section{Perceptual Effect of Shoulder Motions in Crowd Scenarios}

The purpose of this second experiment is to evaluate the effect of adding shoulder motions on the visual quality of crowd animations. To this end, we generate crowd animations which incorporate shoulder motions for characters brushing past each other or having residual collisions. A large difference with the previously studied situation is that only a subset of the visible pairs of characters pass at close distances and actually require to display reactions. As a result, the addition of shoulder motions makes a more subtle visual difference in the context of crowds than in the one of isolated characters. The question guiding the design of this experiment is then about evaluating what remains of the previously observed positive effects, which were particularly strong, now that shoulder motions are diluted into a more complex animation.

\subsection{System description}

2D Global Trajectories. We first generated character global trajectories (first step of a common crowd animation pipeline) using a gradient-based approach in which characters move so as to locally optimize a cost function, similar to [Treuille et al. 2006]. We first played on the initial interpersonal distance between characters to generate stimuli of varying density. The initial position and goal of characters depended on the simulated scenario (described below). In order to evaluate the effect of shoulder motions on the naturalness of residual collisions, we expected trajectories to incorporate a number of collisions. As gradient-based approaches do not explicitly solve for collisions, it was easy to generate residual collisions with a spectrum of interpenetration distances by manually playing on the parameters of the cost function. Table 2 summarises the number of actual collisions between characters, while Figure 8 displays the distribution of interpersonal distances.

Animation Step. Full-body animation of the characters was then performed using motion-capture-based finite state machines (FSMs). We designed a specific FSM per actor using motions from the previous experiment, including a walking motion clip and shoulder motion clips on both the left and right sides (to create additional variety, original left and right motion clips were mirrored and added to the clips of the opposite side).

We designed a simple technique taking advantage of these FSMs to trigger full-body shoulder reactions whenever two characters were passing very close from each other, which therefore preserves synchrony between lower and upper body. As FSMs often include delays because of the blending period between two motions, we computed the expected time and distance to the closest approach (i.e. potential collision) $t t c a$ and $d t c a$ between two characters as:

$$
\begin{aligned}
t t c a_{a_{i}, a_{j}} & =\left\{\begin{array}{cl}
-\frac{\mathbf{p}_{a_{j} \mid a_{i}} \cdot \mathbf{v}_{a_{j} \mid a_{i}}}{\left\|\mathbf{v}_{a_{j} \mid a_{i}}\right\|^{2}} & \text { if }\left\|\mathbf{v}_{a_{j} \mid a_{i}}\right\| \neq 0 \\
\infty & \text { otherwise }
\end{array}\right. \\
d t c a_{a_{i}, a_{j}} & =\left\|\mathbf{p}_{a_{j} \mid a_{i}}+t t c a_{a_{i}, a_{j}} \mathbf{v}_{a_{j} \mid a_{i}}\right\|
\end{aligned}
$$

where $\mathbf{p}_{a_{j} \mid a_{i}}$ and $\mathbf{v}_{a_{j} \mid a_{i}}$ are the current position and velocity of character $j$ relative to character $i$ (2D vectors).

Our algorithm triggers shoulder reactions from the FSM whenever $t t c a<t t c a_{t h}$ and $d t c a<d t c a_{t h}$, where $t t c a_{t h}$ and $d t c a_{t h}$ are parameters of the simulation. We set $d t c a_{t h}$ to $0.6 \mathrm{~m}$, which corresponds to the $5 \%$ perceived collision threshold when no character display a shoulder motion in two-character interactions (Figure 5), and experimentally set $t t c a_{t h}$ to $0.7 \mathrm{~s}$ based on our FSMs. We also triggered appropriate left or right shoulder reactions depending on the side characters would be passing by. Finally, we were also interested in the proportion of characters that should display shoulder motions, as we expected unnatural crowd flows if too many characters were displaying such reactions. We therefore included for each agent a probability $p$ of triggering shoulder motions. Assigning $p$ per agent has also the advantage of representing statistically that either none, one or both characters displayed shoulder motions.

\subsection{Evaluation}

\subsubsection{Factors}

Scenario. We generated human trajectories for two scenarios (Figure 7, Left):

A. A single character walking against a unidirectional crowd flow, where participants were asked to focus on the movements of the single character in relation to the crowd. It is representative of a common video-game gameplay where users follow a main character through a large crowd.

B. Two large groups of characters walking in a bidirectional flow, where participants were asked to focus on the movements in the crowd. It is representative of a common urban scenario.

Density. For each scenario, we controlled the simulator's number of characters and initial distance between agents to create flows with different densities, ranging from sparse to extremely crowded scenes (Figure 7, Right). We selected 5 initial distances (from $0.6 \mathrm{~m}$ to $1.4 \mathrm{~m}$, by $0.2 \mathrm{~m}$ step), resulting in 5 Density levels: $2.8,1.6,1.0$, 0.7 and 0.5 (in characters per square meter $c / \mathrm{m}^{2}$ ). We also created more variety by generating 3 sets of trajectories (repetitions) for each density level using different random initialisations.

Trigger Level. As real humans do not necessarily always display shoulder motions in crowds, we also used 4 levels of probability $p$ of triggering shoulder avoidance reactions: $0 \%, 33 \%, 66 \%$ and $100 \%$. For instance, at the $66 \%$ trigger level characters were triggering a shoulder reaction in $66 \%$ of the cases when an upcoming close interaction was detected.

\subsubsection{Apparatus and Procedure}

Participants were presented in random order with a total of 120 video clips: 2 Scenario $(\mathrm{A}, \mathrm{B}) \times 5$ Density $(0.5,0.7,1.0,1.6$ and $2.8) \times 4$ Trigger Level $(0 \%, 33 \%, 66 \%$ and $100 \%) \times 3$ repetitions. Each video clip lasted 10s. Participants were comfortably seated at their preferred distance from a 24-inch screen, and all the videos were displayed at $1920 \times 1200$ pixels and at $30 \mathrm{~Hz}$ using Psychophysics Toolbox v3 for Matlab. Either Scenario A or B was randomly selected to be shown first, and videos were presented in random order in each block. To get used to the stimuli, six examples that were not seen afterwards in the experiment were presented in random order to participants at the beginning of each block: 3 Density $(0.5,1.0,2.8) \times 2$ Trigger Level $(0 \%, 100 \%)$. To create variety in the crowd appearance, we used 19 female and 20 male models, with 6 different texture variations per model. We were interested in both the perception of collisions and the naturalness of animations, 

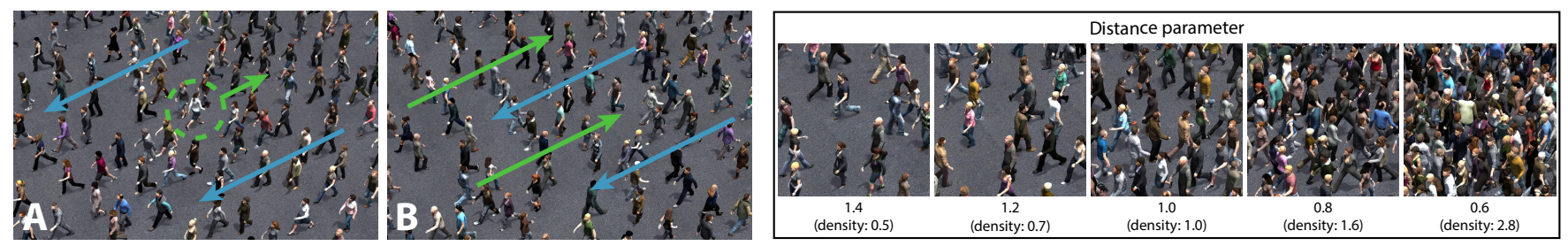

Figure 7: Left: Scenarios of the experiment (A) a single character walking against a unidirectional crowd flow and (B) 2 large groups of characters walking in a bidirectional flow. Right: Levels of densities used in the experiment, controlled by the inter-agent distance parameter.

and therefore asked participants to answer the following two questions: 1) "How often did you see collisions between characters?" (from 1: never to 7: very often) and 2) "How natural was the way the characters moved in the crowd?" (from 1: not very natural to 7: very natural). Participants gave their answers using the number keys on the top row of the keyboard.

Eighteen participants volunteered for this experiment $(6 \mathrm{~F}, 12 \mathrm{M}$; avg. age: $36.1 \pm 9.3$, range: 24 to 54 ). They were recruited via internal mailing lists amongst students and staff, and were all naïve with respect to the purpose of the experiment. Only two participants were familiar with the topic of crowd simulation. All had normal or corrected-to-normal vision. They gave written and informed consent and the study conformed to the declaration of Helsinki.

\subsubsection{Analysis}

Two dependent variables were considered: the collision and the naturalness ratings of the simulation. We evaluated these variables by computing the average ratings over repetitions for each participant. To evaluate the effect of secondary shoulder motions in crowd flows on collision and naturalness ratings, we performed two separated three-way repeated measures ANOVA on the following factors: Scenario $(\mathrm{A}, \mathrm{B}) \times$ Density $(0.5,0.7,1.0,1.6$ and 2.8$) \times$ Trigger Level $(0 \%, 33 \%, 66 \%$ and $100 \%)$. Significant effects from the statistical analyses are summarized in Tables 3.F and 3.G.

\subsection{Results}

There were main and interaction effects of Trigger Level for naturalness ratings. Results showed that naturalness ratings were on average significantly higher when shoulder reactions were presented. More precisely, any amount of shoulder motions (Trigger $>0 \%$ ) improved naturalness for densities over $1.6 \mathrm{c} / \mathrm{m}^{2}$, but only the $100 \%$ level improved naturalness for the $1.0 \mathrm{c} / \mathrm{m}^{2}$ density (Figure 9). Lower densities were not affected by any amount of shoulder motions. This is not surprising since in these cases naturalness ratings are generally high, certainly due to the relatively small number of collisions (Table 2) and the Density $\times$ Scenario interaction effect on collision ratings (Figure 10, Left).

\begin{tabular}{lccccc} 
Density & 0.5 & 0.7 & 1.0 & 1.6 & 2.8 \\
\hline Scenario A & $50 \pm 4$ & $121 \pm 9$ & $305 \pm 11$ & $1140 \pm 51$ & $4546 \pm 76$ \\
\hline Scenario B & $28 \pm 6$ & $97 \pm 12$ & $738 \pm 100$ & $5336 \pm 257$ & $26060 \pm 837$ \\
\hline
\end{tabular}

Table 2: Average $( \pm S D)$ number of collisions detected by our system for each Scenario $\times$ Density condition across repetitions.

There was a main effect of Scenario on both collision and naturalness ratings, where on average less collisions were perceived in Scenario B (i.e., bidirectional flow), which was also rated to be more natural. We also found a main effect of Density in both cases, as well as a Density $\times$ Scenario interaction effect. As expected, this demonstrated that collisions ratings increase and naturalness ratings decrease with increasing densities. Post-hoc analysis showed that

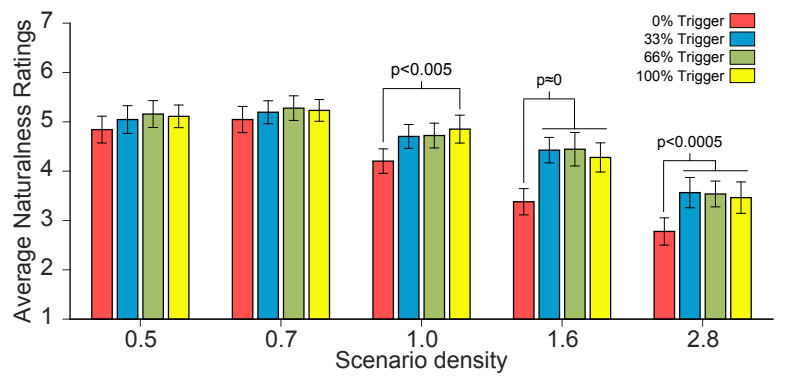

Figure 9: Average naturalness ratings ( \pm SEM) over all participants and both scenarios for each Density $\times$ Trigger condition.
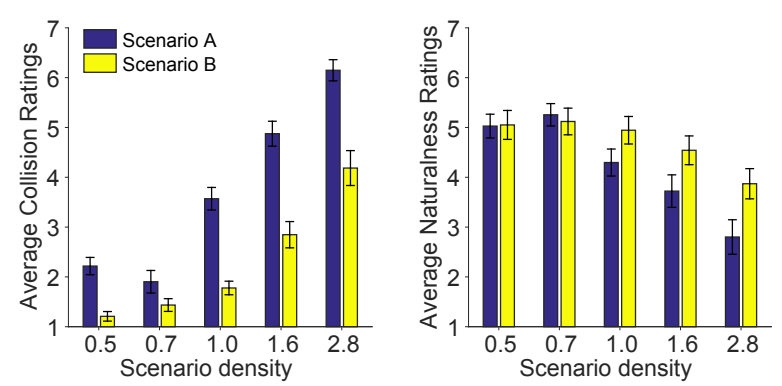

Figure 10: Average collision and naturalness ratings ( \pm SEM) over all participants for each Density $\times$ Scenario condition.

collisions ratings started to significantly increase starting from density 1.0 in Scenario A, while naturalness ratings significantly started to decrease. For Scenario B, collisions ratings started to significantly increase starting from density 1.6 , while naturalness ratings were significantly lower only for density 2.8 (Figure 10).

\subsection{Discussion}

We designed a simple algorithm to trigger shoulder motions in large-scale situations. We studied the effect of triggering various proportions of shoulder motions on the visual quality of resulting animations. As hypothesised, we demonstrated that such secondary motions overall improved the perceived naturalness of animations. This is a remarkable result since, in comparison with the first study, shoulder motions contribute much less to the overall animation: shoulder motions are triggered sparsely in space and time and concern few characters in proportion to all the visible ones, as it can be seen in the companion video. Moreover, our triggering technique introduces low computational overheads, and does not fundamentally change the nature of the animation technique which remains based on finite state machines.

Some factors, such as low densities, seem to limit the benefits of displaying shoulder motions since shoulder motions have no effect on naturalness in these cases. Obviously, density dictates interper- 

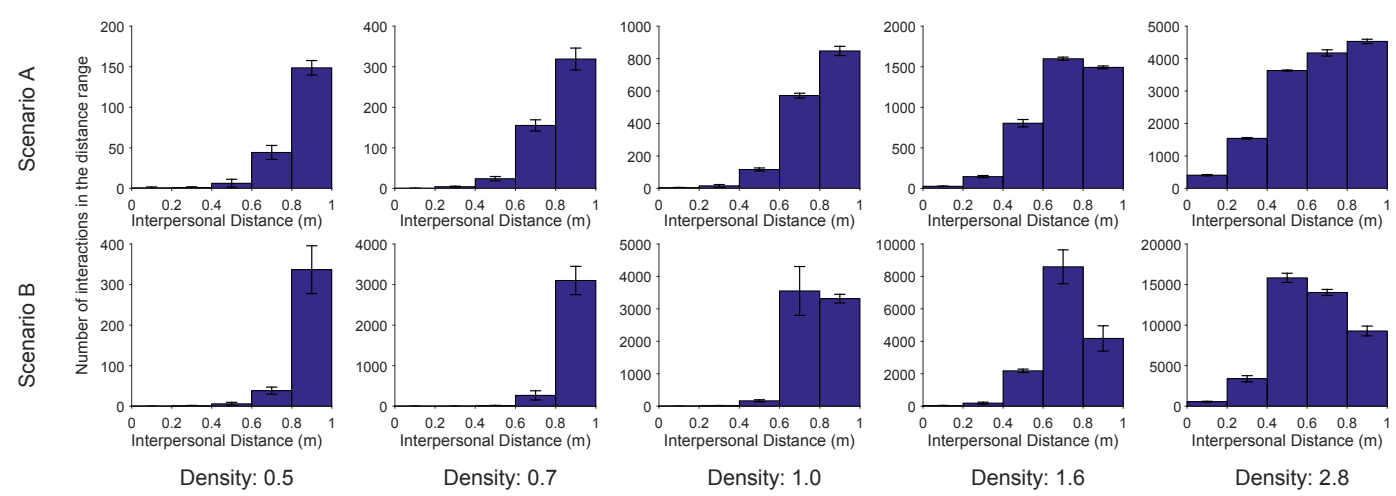

Figure 8: Distribution of interpersonal distances for the sets of trajectories generated for the experiment.

sonal distances. Low densities, i.e., high interpersonal distances, led to only occasional triggering of shoulder motions which might not contribute to a sufficient extent to the overall naturalness of the scene. On the contrary, the highest densities we studied may have led to interpersonal distances that were judged to be visually unrealistic, no matter the presence of shoulder motions.

Interestingly, we expected situations to look less natural when character pairs were always displaying shoulder motions (100\% trigger level), which was never found to be the case. In contrast, this was the only condition found to improve the naturalness of mediumdensity situations. For this experiment, we manually selected a range of shoulder motion probabilities that might not exactly represent how humans behave. Therefore, further studies would be required to improve the calibration of these triggering rules to generate situations as realistic as possible.

Finally, shoulder motions did not affect collision ratings. We remind that interpersonal distances were not explicitly controlled in this experiment, and that some stimuli displayed interactions with large interpenetrations, over the $D_{\text {crit }}$ value estimated in the first experiment. Therefore, it is possible that shoulder motions may not have been sufficient to counterbalance the amount of perceived collisions. We however found differences between the perception of collisions between the two scenarios, where viewers perceived more collisions in Scenario A than B for all the densities (except 0.7 ). Viewers could have more easily perceived the local interactions around the single character in Scenario A, while larger collisions could more easily be missed in Scenario B. The fact that collision ratings increase differently with density between scenarios makes us suppose that viewers may evaluate indirectly the quantity of collisions that should be happening in global situations such as Scenario B, rather than looking directly at the local interactions between characters. This could explain why shoulder motions did not decrease perceived collisions, if information picked by viewers from the crowd density was stronger that the information picked from such secondary motions. A last hypothesis is that detecting collisions in large-scale situations is not a usual task for viewers, as humans rather display contacts than large collisions. Such largescale scenarios can be extremely visually complex and might also include other factors influencing viewers in their judgements, even when the stimuli creation is controlled as much as possible.

\section{General Discussion}

Shoulder motions: difference of effect amplitude between the first and the second experiments. In this paper, we studied the effect of adding shoulder motions on the visual quality of animations, where quality is defined using the perception of residual collisions and of motion naturalness. We considered two situations: 1) with two isolated characters, and 2) with large-scale crowd situations. Our results show that the expected effect is particularly strong in situation 1 , where shoulder motions both increase naturalness and decrease perceived collisions. The effect is attenuated in situation 2 , especially concerning the perception of residual collisions. This attenuation could be a consequence of the large amount of visual information arising from the global flow, which decreases the relative importance of local information such as shoulder motions. In contrast, the importance of shoulder motions is much higher in situation 1, the "worst case scenario", where viewers focus on the two interacting characters and therefore should be the most critical about the quality of animations (i.e., naturalness and collisions). In situation 2, shoulder motions are disseminated in space and time among several characters. Because of the scenes' complexity, other factors may also have influenced viewers in their judgements, even if we tried to control such factors. Therefore, we cannot expect the same amplitude of effect in the results of situation 2. Nevertheless, we still observe a significant effect of adding shoulder motions on the perceived naturalness of situations involving large numbers of characters, at the cost of a very light computational overhead. More surprising is the fact that the perception of residual collisions and motion naturalness are more strongly correlated in situation 1 than in situation 2. Our interpretation is that in situation 2, the presence of residual collisions is also conveyed through the average displayed density and the spatial distribution of characters for a given density. Some comments by participants also led us to think that they may have perceived that the highest displayed levels of density would have made collisions between characters unavoidable.

Effect of collision questions on naturalness ratings. As participants were asked about both collisions and naturalness, it is possible that collision answers affected naturalness ratings. While we expected a negative correlation, we introduced the naturalness questions to control that participants understood the questions about collisions (as naturalness is more general than interpenetration). Asking these two questions also allowed us to evaluate how much the absence of perceived collisions contributes to naturalness, and if some non-colliding situations were considered unnatural. However, the effect of collisions on naturalness seems to have been stronger in the first experiment, as demonstrated by the correlation coefficient, while the large number of factors influencing the realism of the scene might have made judging the naturalness more complex in the second experiment. Future studies could collect separately naturalness ratings, which would be valuable to understand the extent to which naturalness can be affected by focusing on collisions.

Do our technique and results apply to any kind of crowd simulation algorithm? The presented shoulder triggering rules are applied at the animation level, on top of 2D global trajectories, whatever the mean by which trajectories are generated. Therefore, 
our technique and results apply to any crowd simulation algorithm that is able to generate trajectories for individual characters. However, the typical exploitation of our results is to adjust the minimum possible interpersonal distance between characters so as to remain below the critical detection threshold we determined. Some algorithms enable direct control of this parameter (such as any velocitybased simulation technique) and can therefore directly incorporate the results of our study.

Triggering rules and animation system. While using shoulders is obviously a good strategy to avoid obstacles passing on the side (as in our experiments), triggering rules would however need to be extended to naturally avoid obstacles under other directions. For instance, the relevance of triggering shoulder motions could be checked depending on estimated future closest relative positions between agents. Other kind of strategies might also be necessary to prevent collisions in some cases, such as modifying stride length to avoid obstacles passing in front. Also, while our techniques can react to future collisions on both sides simultaneously (reacting on the side with the earliest collision risk decreases the occupied width and proves efficient for the second collision as well), more complex strategies might be depicted by real humans and could be integrated. While our approach was based on finite state machines using full-body motions (which preserved synchrony between lower and upper bodies), studying coordination between upper and lower body motions would also provide valuable insights to further improve the realism of animations.

Other factors. Obvious practical reasons prevented us from increasing the number of factors in our experiments, which led us for instance to use a fixed camera viewpoint. This viewpoint was chosen to make collisions between characters as visible as possible, even though we cannot ensure that residual collisions were always visible. While the camera viewpoint could have an effect on critical threshold values, we still expect shoulder motions to improve these thresholds. We also do not take into account whether the interpenetration is visible on screen or hidden by some character body parts, which could be relevant to speed-up collision avoidance in the future. Finally, while we only used motions from four male actors, we believe that they provided enough variety as we did not focus on specific individual motions. We therefore expect similar results when using other characters, or female motions, which could be validated in the future. None of the participants mentioned that they considered motions of female characters to look unnatural in the second experiment, even though we only used motions of two male actors. The contribution of using gender-specific motions to the overall naturalness of the scene could however be further explored in the future, especially as previous work found that male and female motions can be perceived as ambiguous when displayed on characters of incongruent sex [McDonnell et al. 2009].

\section{Conclusion}

In this paper we studied the effect of adding shoulder motions on the animation of crowd characters. In a first experiment, we considered isolated pairs of characters and demonstrated that shoulder motions significantly improve the visual quality of animations. This improvement is revealed through our measurements of the perception of residual collisions and the evaluation of motion naturalness. In a second experiment, we considered flows of crowd characters, and demonstrated that the effect on the naturalness of animations remains significant, while the effect on the perception of residual collisions is attenuated. Nevertheless, the trade-off between the cost of adding shoulder motions and the computation cost of such additional objectively is a good one.

More generally, our work highlights the benefit of adding secondary motions to procedurally animated characters. Previous techniques allow to introduce motion variety when animating moving characters (by modulating template procedural animations), or to animate still characters during interactions (e.g., chatting characters). Our work opens a new path: we add variations to the motions of walking characters by adding interaction-dependent secondary motions at very low computational overheads. Such additions do not only improve how characters move, but also how they move with respect to their direct neighbours.

Whereas our work focused on shoulder motions, we believe that many other kind of secondary motions which result from interactions could be considered, such as forms of social graces for characters politely giving way, expressions of surprise for characters close to colliding, eye-contacts for characters negotiating avoidance, etc. More generally, a promising direction for future work is to store some useful information on ongoing interactions at the stage of simulation, and to re-use it at the stage of animation. Indeed, crowd simulators already compute various types of spatio-temporal information about ongoing character interactions (e.g., distances, relative positions or velocities, time to collision). This data is used to compute how each character's trajectory is influenced by neighbours but discarded at the stage of animation, whereas it could also be used to trigger specific character animations.

\section{Acknowledgements}

We wish to thank all the reviewers for their constructive comments, as well as the participants who took part in our experiments. This work was funded by the ANR-13-JS02-0008 Percolation project.

\section{References}

Ennis, C., AND O'Sullivan, C. 2012. Perceptually plausible formations for virtual conversers. Computer Animation and Virtual Worlds 23, 3-4, 321-329.

Ennis, C., McDonnell, R., And O'Sullivan, C. 2010. Seeing is believing: Body motion dominates in multisensory conversations. ACM Trans. Graph. 29, 4 (July), 91:1-91:9.

Helbing, D., And MolnáR, P. 1995. Social force model for pedestrian dynamics. Physical Review E 51, 5, 4282-4286.

Hoyet, L., McDonnell, R., And O’Sullivan, C. 2012. Push it real: Perceiving causality in virtual interactions. ACM Trans. Graph. 31, 4 (July), 90:1-90:9.

Hughes, R., OndřEJ, J., AND Dingliana, J. 2014. Holonomic collision avoidance for virtual crowds. In Proceedings of the Symposium on Computer Animation, 103-111.

Hughes, R. L. 2003. The flow of human crowds. Annual review of fluid mechanics 35, 1, 169-182.

Karamouzas, I., Skinner, B., AND GUY, S. J. 2014. Universal power law governing pedestrian interactions. Phys. Rev. Lett. 113 (Dec), 238701.

Kim, M., Hwang, Y., Hyun, K., And Lee, J. 2012. Tiling motion patches. In Proceedings of the Symposium on Computer Animation, 117-126.

Korhonen, T., HeliövaAra, S., Ehtamo, H., AND HostikKa, S. 2009. Collision avoidance and shoulder rotation in pedestrian modeling. Tech. rep., Aalto University.

Kulpa, R., Olivier, A.-H., Ondřej, J., And Pettré, J. 2011. Imperceptible relaxation of collision avoidance constraints in virtual crowds. ACM Trans. Graph. 30, 6 (Dec.), 138:1-138:10. 
Perceptual EfFect of Shoulder Motions in Two-Character InTERaCtions

\begin{tabular}{|c|c|c|}
\hline \multicolumn{3}{|c|}{ A. Perceived Collisions vs. Interpersonal Distance -3 Shoulder Motion $(\mathrm{MOTION}) \times 11$ Interpersonal Distance $(\mathrm{DIST})$} \\
\hline Effect & F-Test & Post-hoc \\
\hline MOTION & $F_{(2,28)}=1082.4, p \approx 0, \eta_{p}^{2}=0.99$ & Less collisions perceived when 2 characters display shoulder motions, then 1 , then 0 \\
\hline DIST & $F_{(10,140)}=923.5, p \approx 0, \eta_{p}^{2}=0.99$ & Less collisions perceived when the distance between characters increases \\
\hline MOTION $\times$ DIST & $F_{(20,280)}=127.9, p \approx 0, \eta_{p}^{2}=0.90$ & Range of distances where collisions are perceived differ between MOTION conditions \\
\hline \multicolumn{3}{|c|}{ B. Critical Distance Threshold $\mathbf{D}_{\text {crit }}-3$ Shoulder Motion (MOTION) } \\
\hline Effect & F-Test & Post-hoc \\
\hline MOTION & $F_{(1.4,20)}=489.2, p \approx 0, \eta_{p}^{2}=0.97$ & $D_{\text {crit }}$ lower when 2 characters display shoulder motions, then 1 , then 0 \\
\hline \multicolumn{3}{|c|}{ C. Critical Volume Threshold $\mathbf{V}_{\text {crit }}-3$ Shoulder Motion (MOTION) } \\
\hline Effect & F-Test & Post-hoc \\
\hline MOTION & $F_{(2,28)}=137.94, p \approx 0, \eta_{p}^{2}=0.90$ & $V_{\text {crit }}$ higher when 2 characters display shoulder motions, then 1 , then 0 \\
\hline \multicolumn{3}{|c|}{ D. Naturalness vs. Interpersonal Distance -3 Shoulder Motion $(\mathrm{MOTION}) \times 11$ Interpersonal Distance (DIST) } \\
\hline Effect & F-Test & Post-hoc \\
\hline MOTION & $F_{(2,28)}=96.45, p \approx 0, \eta_{p}^{2}=0.87$ & Higher average naturalness ratings when 2 characters display shoulder motions, then 1 , then 0 \\
\hline DIST & $F_{(2.0,28.4)}=140.30, p \approx 0, \eta_{p}^{2}=0.91$ & Average naturalness ratings increase with interpersonal distance \\
\hline MOTION $\times$ DIST & $F_{(20,280)}=42.97, p \approx 0, \eta_{p}^{2}=0.75$ & High naturalness for smaller distances when 2 characters display shoulder motions, then 1 , then 0 \\
\hline \multicolumn{3}{|c|}{ E. Naturalness vs. Interpenetration Volume (Slope of linear regression) - 3 Shoulder Motion (MOTION) } \\
\hline Effect & F-Test & Post-hoc \\
\hline MOTION & $F_{(2,28)}=53.73, p \approx 0, \eta_{p}^{2}=0.79$ & Slower naturalness decrease with interpenetration volume for Shoulder Motion 2, then 1 , then 0 \\
\hline \multicolumn{3}{|c|}{ PERCEPTUAL EFFECT OF ShOUlder MOTIONS IN CROWD SCENARIOS } \\
\hline \multicolumn{3}{|c|}{ F. Collision Ratings -2 Scenario $($ SCE) $\times 5$ Density $(\mathrm{DEN}) \times 4$ Trigger Level $(\mathrm{TRI})$} \\
\hline Effect & F-Test & Post-hoc \\
\hline SCE & $F_{(1,17)}=108.70, p \approx 0, \eta_{p}^{2}=0.86$ & Less collisions perceived in Scenario B than A \\
\hline DEN & $F_{(2.1,35.7)}=242.33, p \approx 0, \eta_{p}^{2}=0.93$ & Less collisions perceived for densities 0.5 and 0.7 , then 1.0 , then 1.6 and 2.8 . \\
\hline $\mathrm{SCE} \times \mathrm{DEN}$ & $F_{(1.8,30.5)}=14.09, p \approx 0, \eta_{p}^{2}=0.45$ & More perceived collisions starting from density 1.0 (Sce. A), and starting from density 1.6 (Sce. B) \\
\hline \multicolumn{3}{|c|}{ G. Naturalness Ratings -2 Scenario $(\mathrm{SCE}) \times 5$ Density $(\mathrm{DEN}) \times 4$ Trigger Level $(\mathrm{TRI})$} \\
\hline Effect & F-Test & Post-hoc \\
\hline SCE & $F_{(1,17)}=5.23, p<0.05, \eta_{p}^{2}=0.24$ & Higher naturalness ratings for Scenario B than A \\
\hline DEN & $F_{(1.7,29.1)}=36.45, p \approx 0, \eta_{p}^{2}=0.68$ & Naturalness ratings decrease with density \\
\hline TRI & $F_{(1.5,25.3)}=15.85, p \approx 0, \eta_{p}^{2}=0.48$ & No shoulder motion (Trigger Level $0 \%$ ) less natural than everything else \\
\hline SCE $\times$ DEN & $F_{(2.2,37.0)}=7.90, p<0.00005, \eta_{p}^{2}=0.32$ & Densities 0.5 and 0.7 as natural for both scenarios, others less natural for Scenario A than B \\
\hline SCE $\times$ TRI & $F_{(3,51)}=4.63, p<0.01, \eta_{p}^{2}=0.21$ & Trigger Level $0 \%$ less natural than everything else in A and B, all other levels less natural in A than B \\
\hline DEN $\times$ TRI & $F_{(12,204)}=3.23, p<0.0005, \eta_{p}^{2}=0.16$ & Trigger Level $0 \%$ less natural than other levels for densities $1.0,1.6$ and 2.8 \\
\hline
\end{tabular}

Table 3: Main significant results of the presented experiments.

Kwon, T., And Shin, S. Y. 2005. Motion modeling for online locomotion synthesis. In Proceedings of the Symposium on Computer Animation, 29-38.

Lee, J., Chai, J., Reitsma, P., Hodgins, J., And Pollard, N. 2002. Interactive control of avatars animated with human motion data. ACM Trans. Graph. 21, 3 (July), 491-500.

MAÏM, J., Yersin, B., AND ThALMANN, D. 2009. Unique character instances for crowds. IEEE Computer Graphics and Applications, 6, 82-90.

McDonnell, R., Larkin, M., Dobbyn, S., Collins, S., AND O'Sullivan, C. 2008. Clone attack! perception of crowd variety. ACM Trans. Graph. 27, 3 (Aug.), 26:1-26:8.

McDonnell, R., JÖRG, S., Hodgins, J., Newell, F., AND O'Sullivan, C. 2009. Evaluating the effect of motion and body shape on the perceived sex of virtual characters. $A C M$ Trans. Appl. Percept. 5, 4 (Feb.), 20:1-20:14.

OndŘEJ, J., Pettré, J., Olivier, A.-H., And Donikian, S. 2010. A synthetic-vision based steering approach for crowd simulation. ACM Trans. Graph. 29, 4 (July), 123:1-123:9.

Reitsma, P. S. A., And O'Sullivan, C. 2009. Effect of scenario on perceptual sensitivity to errors in animation. $A C M$ Trans. Appl. Percept. 6, 3 (Sept.), 15:1-15:16.

REYNOLDS, C. 1987. Flocks, herds and schools: A distributed behavioral model. SIGGRAPH Comp. Graphics 21, 4, 25-34.

Rose, C., Cohen, M. F., And Bodenheimer, B. 1998. Verbs and adverbs: Multidimensional motion interpolation. IEEE Comput. Graph. Appl. 18, 5 (Sept.), 32-40.
Shum, H. P. H., Komura, T., Shiraishi, M., AND YamaZaKi, S. 2008. Interaction patches for multi-character animation. ACM Trans. Graph. 27, 5 (Dec.), 114:1-114:8.

Singh, S., Kapadia, M., Reinman, G., and Faloutsos, P. 2011. Footstep navigation for dynamic crowds. Computer Animation and Virtual Worlds 22, 2-3, 151-158.

Stüvel, S. A., Magnenat-Thalmann, N., Thalmann, D., EgGes, A., AND VAN DER STAPPEN, A. F. 2014. Hierarchical structures for collision checking between virtual characters. Computer Animation and Virtual Worlds 25, 3-4, 331-340.

StÜVel, Magnenat-Thalmann, N., Thalmann, D., VAN DER Stappen, A., AND EgGes, A. 2016. Torso crowds. IEEE Transactions on Visualization and Computer Graphics, 99.

Thalmann, D. 2007. Crowd simulation. Wiley Online Library.

Treuille, A., Cooper, S., ANd Popović, Z. 2006. Continuum crowds. ACM Trans. Graph. 25, 3 (July), 1160-1168.

Treuille, A., Lee, Y., AND Popović, Z. 2007. Nearoptimal character animation with continuous control. ACM Trans. Graph. 26, 3 (July).

VAN DEN BerG, J., LIN, M., AND MANocha, D. 2008. Reciprocal velocity obstacles for real-time multi-agent navigation. In IEEE Int. Conf. on Robotics and Automation, 1928-1935.

Yeh, T., Reinman, G., Patel, S., and Faloutsos, P. 2009. Fool me twice: Exploring and exploiting error tolerance in physics-based animation. ACM Trans. Graph. 29, 1, 5:1-5:11. 